\title{
ASYMPTOTIC NORMALITY OF DEGREE COUNTS IN A PREFERENTIAL ATTACHMENT MODEL
}

\author{
SIDNEY I. RESNICK AND GENNADY SAMORODNITSKY
}

\begin{abstract}
Preferential attachment is a widely adopted paradigm for understanding the dynamics of social networks. Formal statistical inference, for instance GLM techniques, and model verification methods will require knowing test statistics are asymptotically normal even though node or count based network data is nothing like classical data from independently replicated experiments. We therefore study asymptotic normality of degree counts for a sequence of growing simple undirected preferential attachment graphs. The methods of proof rely on identifying martingales and then exploiting the martingale central limit theorems.
\end{abstract}

\section{INTRODUCTION}

Preferential attachment is a widely adopted model for understanding social network growth. The assumption posits that nodes with a large number of existing connections are more likely to attract connections from new nodes joining the network. This paradigm is one of the justifications for perceived power law behavior.

Statistical analyses of social networks is complicated by the fact that node based data is nothing like classical iid data obtained from repeated sampling. Efforts at statistical estimation and model confirmation center around graphical based slope methods, regression models for the center of the data and tail estimation methodology. These statistical techniques are adaptations of classical methods but currently are largely without justification. Yet they produce reasonable answers and plots.

We begin a program for justifying statistical methodology by examining the asymptotic normality of counting variables for the number of nodes of degree $k$ in a simple undirected preferential attachment model described in [11, Chapter 8]. We let $N_{n}(k)$ be the number of nodes of degree $k$ at the $n$th stage of development of the network. It is known $N_{n}(k) / n \rightarrow p_{k}$ and for our model, the sequence $\left\{p_{k}\right\}$ can be exhibited explicitly. The martingale central limit theorem allows us to prove asymptotic normality for $\sqrt{n}\left(N_{k}(k) / n-p_{k}\right)$. This emphasizes consistency of the empirical count percentages as estimates of $\left\{p_{k}\right\}$ and provides confidence statements for the estimates.

2010 Mathematics Subject Classification. 28A33,60G17,60G51,60G70.

Key words and phrases. power law, degree counts, preferential attachment, random graphs.

S. Resnick and G. Samorodnitsky were supported by Army MURI grant W911NF-12-1-0385 to Cornell University. 
We describe the undirected preferential attachment model in Section 1.1 and give known mathematical results that we need in Section 3 Asymptotic normality is considered in the simplest case $k=1$ in Section 3 and for the general case in Section 4

1.1. The model for preferential attachment. We consider a simple growing undirected graph with preferential attachment that is outlined in 11, Chapter 8] or [5]. The random graph at stage $n$ is $G_{n}=\left(V_{n}, E_{n}\right)$, the set of nodes or vertices is $V_{n}:=\{1, \ldots, n\}$ and the set of undirected edges is $E_{n}$, a subset of $\left\{\{i, j\}: i, j \in V_{n}\right\}$. For $v \in V_{n}$, let $D_{n}(v)$ be the degree of $v$ at stage $n$; that is, the number of edges incident to $v$. As a convenient and harmless initialization, assume $V_{1}$ consists of a single node 1 with a self-loop so that $D_{1}(1)=2$.

Conditional on knowing the graph $G_{n}$, at stage $n+1$ a new node $n+1$ appears and with a parameter $\delta>-1$, either

(1) The new node $n+1$ attaches to $v \in V_{n}$ with probability

$$
\frac{D_{n}(v)+\delta}{n(2+\delta)+(1+\delta)}
$$

or

(2) $n+1$ attaches to itself with probability

$$
\frac{1+\delta}{n(2+\delta)+(1+\delta)}
$$

In the first case $D_{n+1}(n+1)=1$ and in the second case $D_{n+1}(n+1)=2$. It is standard for this model that

$$
\sum_{v \in V_{n}} D_{n}(v)=2 n
$$

and thus the attachment probabilities in (1.1) and (1.2) add to 1.

\section{Martingale Central Limit theorem}

Martingale central limit theorems have been used for a long time. In order to make the paper self-contained, we present the statements we will need in this section. We start with a one-dimensional martingale adaptation of the LindebergFeller central limit theorem ([4, Chapter 8] or [6] ).

Proposition 2.1. Let $\left\{X_{n, m}, \mathcal{F}_{n, m}, 1 \leqslant m \leqslant n\right\}$ be a square integrable martingale difference array satisfying

(1) $V_{n, n}:=\sum_{m \leqslant n} E\left(X_{n, m}^{2} \mid \mathcal{F}_{n, m-1}\right) \stackrel{P}{\rightarrow} \sigma^{2}$ as $n \rightarrow \infty$.

(2) $\sum_{m \leqslant n} E\left(X_{n, m}^{2} 1_{\left[\left|X_{n, m}\right|>\epsilon\right]} \mid \mathcal{F}_{n, m-1}\right) \stackrel{P}{\rightarrow} 0$ as $n \rightarrow \infty$ for all $\epsilon>0$.

Then as $n \rightarrow \infty$,

$$
\sum_{m=1}^{n} X_{n, m} \Rightarrow N\left(0, \sigma^{2}\right)
$$

Using the Cramér-Wold device, it is not hard to extend the statement of Proposition 2.1 to the multivariate case. 
Proposition 2.2. Let $\left\{\boldsymbol{X}_{n, m}, \mathcal{F}_{n, m}, 1 \leqslant m \leqslant n\right\}, \boldsymbol{X}_{n, m}=\left(X_{n, m, 1}, \ldots, X_{n, m, d}\right)^{T}$, be a d-dimensional square-integrable martingale difference array. Consider the $d \times d$ nonnegative definite random matrices

$$
G_{n, m}=\left(E\left(X_{n, m, i} X_{n, m, j} \mid \mathcal{F}_{n, m-1}\right), i, j=1, \ldots, d\right), \quad V_{n}=\sum_{m=1}^{n} G_{n, m},
$$

and suppose $\left(A_{n}\right)$ is a sequence of $l \times d$ matrices with a bounded supremum norm. Assume that

(1) $A_{n} V_{n} A_{n}^{T} \stackrel{P}{\rightarrow} \Sigma$ as $n \rightarrow \infty$ for some nonrandom (automatically nonnegatively definite) matrix $\Sigma$.

(2) $\sum_{m \leqslant n} E\left(X_{n, m, i}^{2} 1_{\left[\left|X_{n, m, i}\right|>\epsilon\right]} \mid \mathcal{F}_{n, m-1}\right) \stackrel{P}{\rightarrow} 0$ as $n \rightarrow \infty$ for all $i=1, \ldots, d$ and $\epsilon>0$.

Then in $\mathbb{R}^{l}$,

$$
\sum_{m=1}^{n} A_{n} \boldsymbol{X}_{n, m} \Rightarrow \boldsymbol{X}, \quad(n \rightarrow \infty),
$$

a centered l-dimensional Gaussian vector with covariance matrix $\Sigma$.

Proof. By the Cramér-Wold device, it is enough to prove that for any vector $\boldsymbol{b} \in \mathbb{R}^{l}$,

$$
\sum_{m=1}^{n} \boldsymbol{b}^{T} A_{n} \boldsymbol{X}_{n, m} \Rightarrow N\left(0, \boldsymbol{b}^{T} \Sigma \boldsymbol{b}\right) .
$$

Since $\left\{\boldsymbol{b}^{T} A_{n} \boldsymbol{X}_{n, m}, \mathcal{F}_{n, m}, 1 \leqslant m \leqslant n\right\}$ is a one-dimensional square integrable martingale difference array, we only need to check the conditions of Proposition 2.1] Since

$$
\sum_{m \leqslant n} E\left(\left(\boldsymbol{b}^{T} A_{n} \boldsymbol{X}_{n, m}\right)^{2} \mid \mathcal{F}_{n, m-1}\right)=\boldsymbol{b}^{T} A_{n} V_{n} A_{n}^{T} \boldsymbol{b} \stackrel{P}{\rightarrow} \boldsymbol{b}^{T} \Sigma \boldsymbol{b}
$$

as $n \rightarrow \infty$, the first condition of that proposition holds. For the second condition, notice that for every $\epsilon>0$ we can write

$$
\left|\boldsymbol{b}^{T} A_{n} \boldsymbol{X}_{n, m}\right| \leqslant d \max _{i=1, \ldots, d}\left|X_{n, m, i} \sum_{j=1}^{l} b_{j} A_{n}(j, i)\right| \leqslant \theta \max _{i=1, \ldots, d}\left|X_{n, m, i}\right|,
$$

where $\theta$ is a finite positive constant depending on the vector $\boldsymbol{b}$ and an upper bound on the supremum norm of the sequence $\left(A_{n}\right)$. Therefore,

$$
\begin{gathered}
\sum_{m \leqslant n} E\left(\left(\boldsymbol{b}^{T} A_{n} \boldsymbol{X}_{n, m}\right)^{2} 1_{\left|\boldsymbol{b}^{T} A_{n} \boldsymbol{X}_{n, m}\right|>\epsilon} \mid \mathcal{F}_{n, m-1}\right) \\
\leqslant \theta^{2} \sum_{m \leqslant n} E\left(\max _{i=1, \ldots, d}\left|X_{n, m, i}\right|^{2} 1_{\max _{i=1, \ldots, d}\left|X_{n, m, i}\right|>\epsilon / \theta} \mid \mathcal{F}_{n, m-1}\right) \\
\leqslant \theta^{2} \sum_{i=1}^{d} \sum_{m \leqslant n} E\left(X_{n, m, i}^{2} 1_{\left|X_{n, m, i}\right|>\epsilon / \theta} \mid \mathcal{F}_{n, m-1}\right) \rightarrow 0
\end{gathered}
$$

by the second assumption of the present proposition. This verifies the assumptions of Proposition 2.1 and, hence, completes the argument. 


\section{Asymptotic Normality of DEGREe Counts}

For $k \geqslant 1$, let $N_{n}(k)$ be the number of nodes in $G_{n}$ with degree $k$ :

$$
N_{n}(k)=\sum_{v \in V_{n}} 1_{\left[D_{n}(v)=k\right]} .
$$

Using concentration inequalities and martingale methods it is shown, for instance in [11, Chapter 8] that there is a probability mass function $\left\{p_{k}, k \geqslant 1\right\}$ such that almost surely as $n \rightarrow \infty$,

$$
\frac{N_{n}(k)}{n} \rightarrow p_{k}, \quad k \geqslant 1
$$

and

$$
p_{k}=\left(\frac{(2+\delta) \Gamma(3+2 \delta)}{\Gamma(1+\delta)}\right) \frac{\Gamma(k+\delta)}{\Gamma(k+3+2 \delta)}=c(\delta) \frac{\Gamma(k+\delta)}{\Gamma(k+3+2 \delta)} .
$$

Of course $\Gamma(\cdot)$ is the gamma function. In particular, for $k=1$

$$
p_{1}=\frac{2+\delta}{3+2 \delta}
$$

and as $k \rightarrow \infty$, power law behavior is

$$
p_{k} \sim c(\delta) k^{-3-\delta}
$$

We consider the asymptotic normality of $N_{n}(k) / n-p_{k}$ for $k \geqslant 1$, and we start with the simplest case of $k=1$.

When proving the asymptotic normality for the number of nodes of degree 1 , we will use the abbreviations

$$
\nu_{n}=E\left(N_{n}(1)\right), \quad \gamma=\frac{1+\delta}{2+\delta}, \quad w_{n}=\frac{n}{n+\gamma} .
$$

Note $N_{1}(1)=\nu_{1}=0$ since the initial node has a self-loop so $D_{1}(1)=2$.

Let $\mathcal{F}_{n}$ be the information from observing the growth of the network up through the $n$th stage. Then

$$
\begin{aligned}
E\left(N_{n+1}(1) \mid \mathcal{F}_{n}\right)= & N_{n}(1)+E\left(\left(N_{n+1}(1)-N_{n}(1)\right) \mid \mathcal{F}_{n}\right) \\
= & N_{n}(1)+E\left(1_{\left.\left[n+1 \text { links to } v \in V_{n} ; D_{n}(v)>1\right] \mid \mathcal{F}_{n}\right)}\right. \\
= & N_{n}(1)+1-P\left[n+1 \text { links to itself } \mid \mathcal{F}_{n}\right] \\
& \quad-P\left[n+1 \text { links to } v \in V_{n} ; D_{n}(v)=1 \mid \mathcal{F}_{n}\right]
\end{aligned}
$$

and using (1.2) and the fact that for the last term $D_{n}(v)=1$, we get

$$
\begin{aligned}
& =N_{n}(1)+1-\frac{1+\delta}{n(2+\delta)+(1+\delta)}-\frac{1+\delta}{n(2+\delta)+(1+\delta)} N_{n}(1) \\
& =N_{n}(1)\left(1-\frac{\gamma}{n+\gamma}\right)+\left(1-\frac{\gamma}{n+\gamma}\right) \\
& =w_{n} N_{n}(1)+w_{n} .
\end{aligned}
$$

We conclude

$$
E\left(N_{n+1}(1) \mid \mathcal{F}_{n}\right)=w_{n} N_{n}(1)+w_{n}
$$




$$
\nu_{n+1}=w_{n} \nu_{n}+w_{n}
$$

We claim

$$
M_{n+1}:=\frac{N_{n+1}(1)-\nu_{n+1}}{\prod_{j=1}^{n} w_{j}}=\frac{N_{n+1}(1)-\sum_{l=1}^{n} \prod_{j=l}^{n} w_{j}}{\prod_{j=1}^{n} w_{j}}, \quad n \geqslant 1
$$

is a martingale. This is verified using (3.4) and (3.5).

Consider now the martingale difference

$$
\begin{aligned}
d_{n+1} & :=M_{n+1}-M_{n}=\frac{N_{n+1}(1)-\nu_{n+1}}{\prod_{j=1}^{n} w_{j}}-\frac{N_{n}(1)-\nu_{n}}{\prod_{j=1}^{n-1} w_{j}} \\
& =\frac{1}{\prod_{j=1}^{n} w_{j}}\left(N_{n+1}(1)-\nu_{n+1}-\left(w_{n} N_{n}(1)-w_{n} \nu_{n}\right)\right) \\
& =\frac{1}{\prod_{j=1}^{n} w_{j}}\left(N_{n+1}(1)-N_{n}(1)+N_{n}(1)\left(1-w_{n}\right)-w_{n}\right) .
\end{aligned}
$$

As above,

$$
\left.N_{n+1}(1)-N_{n}(1)=: \Delta_{n+1}=1_{[n+1} \text { links with } v \in V_{n} ; D_{n}(v)>1\right],
$$

and $\Delta_{n+1}^{2}=\Delta_{n+1}$. Therefore,

$$
\begin{aligned}
& d_{n+1}^{2}= \frac{1}{\left(\prod_{j=1}^{n} w_{j}\right)^{2}}\left(\Delta_{n+1}+2 \Delta_{n+1}\left(N_{n}(1)\left(1-w_{n}\right)-w_{n}\right)\right. \\
&\left.\quad+\left(N_{n}(1)\left(1-w_{n}\right)-w_{n}\right)^{2}\right) \\
&=\frac{1}{\left(\prod_{j=1}^{n} w_{j}\right)^{2}}\left(\Delta_{n+1}\left\{1+2\left(\left(1-w_{n}\right) N_{n}(1)-w_{n}\right)\right\}\right. \\
&\left.\quad+\left(\left(1-w_{n}\right) N_{n}(1)-w_{n}\right)^{2}\right) .
\end{aligned}
$$

Since

$$
E\left(\Delta_{n+1} \mid \mathcal{F}_{n}\right)=E\left(N_{n+1}(1)-N_{n}(1) \mid \mathcal{F}_{n}\right)=1-\frac{\gamma}{n+\gamma}\left(1+N_{n}(1)\right),
$$

we have

$$
\begin{aligned}
E\left(d_{n+1}^{2} \mid \mathcal{F}_{n}\right)= & \frac{1}{\left(\prod_{j=1}^{n} w_{j}\right)^{2}}\left(\{ 1 + 2 ( ( 1 - w _ { n } ) N _ { n } ( 1 ) - w _ { n } ) \} \left(1-\frac{\gamma}{n+\gamma}\left(1+N_{n}(1)\right)\right.\right. \\
& \left.+\left[\left(1-w_{n}\right) N_{n}(1)-w_{n}\right]^{2}\right) \\
(3.8) \quad= & : \frac{1}{\left(\prod_{j=1}^{n} w_{j}\right)^{2}} \chi_{n} .
\end{aligned}
$$

We need the following observations:

(a) $w_{n}=n /(n+\gamma) \rightarrow 1$, as $n \rightarrow \infty$.

(b) $1-w_{n}=\gamma /(n+\gamma) \sim \gamma / n \rightarrow 0$. 
(c) Owing to the usual recursion on the gamma function,

$$
\frac{\Gamma(n+1+\gamma)}{\Gamma(1+\gamma)}=\prod_{j=0}^{n-1}(n+\gamma-j)=\prod_{l=1}^{n}(\gamma+l)
$$

(d) Therefore we have

$$
\begin{aligned}
\prod_{j=1}^{n} w_{j} & =\prod_{j=1}^{n}\left(\frac{j}{j+\gamma}\right)=\frac{\Gamma(n+1)}{\Gamma(n+1+\gamma)} \Gamma(1+\gamma) \\
& \sim \Gamma(1+\gamma) n^{-\gamma}=\Gamma\left(\frac{1}{p_{1}}\right) n^{-\gamma}
\end{aligned}
$$

by Stirling's formula.

(e) We have

$$
\lim _{n \rightarrow \infty} \frac{N_{n}(1)}{n}=\lim _{n \rightarrow \infty} \frac{\nu_{n}}{n}=p_{1}=\frac{2+\delta}{3+2 \delta} .
$$

This allows us to evaluate $\lim _{n \rightarrow \infty} \chi_{n}$ in (3.8) as

$$
\begin{aligned}
c_{0}(\delta): & \left.=\left(1+2\left(\gamma p_{1}-1\right)\right]\left[1-\gamma p_{1}\right]\right)+\left(\gamma p_{1}-1\right)^{2}=\gamma p_{1}\left(1-\gamma p_{1}\right) \\
& =\left(\frac{1+\delta}{3+2 \delta}\right)\left(\frac{2+\delta}{3+2 \delta}\right)=\frac{(1+\delta)(2+\delta)}{(3+2 \delta)^{2}} .
\end{aligned}
$$

Therefore from (3.9), (3.8) and (3.10)

$$
E\left(d_{n+1}^{2} \mid \mathcal{F}_{n}\right) \sim \frac{c_{0}(\delta)}{\left(\Gamma(1+\gamma) n^{-\gamma}\right)^{2}}=: c_{1}(\delta) n^{2 \gamma}
$$

with

$$
c_{1}(\delta)=c_{0}(\delta) /(\Gamma(1+\gamma))^{2} .
$$

Keeping in mind that the asymptotic equivalence in 3.11 holds a.s, we apply Karamata's theorem on integration (eg, [8, page 25] or [2]), to obtain

$$
\sum_{j=1}^{n} E\left(d_{j+1}^{2} \mid \mathcal{F}_{j}\right) \sim \frac{c_{1}(\delta)}{2 \gamma+1} n^{2 \gamma+1}=: \sigma^{2}(\delta) n^{2 \gamma+1}, \quad(n \rightarrow \infty)
$$

with

$$
\sigma^{2}(\delta)=\frac{(1+\delta)(2+\delta)}{(3+2 \delta)^{2}(2 \gamma+1)(\Gamma(1+\gamma))^{2}}
$$

Now set

$$
d_{n, m}=\frac{d_{m}}{\sigma(\delta) n^{\gamma+1 / 2}}, \quad 1 \leqslant m \leqslant n,
$$

and the first condition of Proposition 2.1] is satisfied. For the second condition of Proposition 2.1 observe that from (3.7), we get by ignoring constants and remembering $\left|\Delta_{m+1}\right| \leqslant 1$, that, as $m \rightarrow \infty$,

$$
\begin{aligned}
{\left[\left|d_{n, m+1}\right|>\epsilon\right] } & =\left[\left|d_{m+1}\right|>\epsilon n^{\gamma+1 / 2}\right] \\
& \subset\left[\frac{1}{\prod_{j=1}^{m} w_{j}} \mid\left[1+N_{m}(1)\left(1-w_{m}\right)-w_{m} \mid>\epsilon n^{\gamma+1 / 2}\right]\right.
\end{aligned}
$$




$$
\begin{aligned}
& \subset\left[c m^{\gamma} \mid(\text { const }) \mid>\epsilon n^{\gamma+1 / 2}\right] \\
& \subset\left[c n^{\gamma} \mid(\text { const }) \mid>\epsilon n^{\gamma+1 / 2}\right] .
\end{aligned}
$$

So with probability converging to 1 as $n \rightarrow \infty$, all the indicator functions $1_{\left[\left|d_{n, m}\right|>\epsilon\right]}$ vanish, and this verifies the second condition for the central limit theorem.

We conclude from Proposition 2.1 that (2.1) holds. Unpacking (2.1), we find

$$
\sum_{m=1}^{n} d_{n, m}=\sum_{m=1}^{n} \frac{d_{m}}{\sigma(\delta) n^{\gamma+1 / 2}}=\frac{1}{\sigma(\delta) n^{\gamma+1 / 2}}\left(\frac{N_{n}(1)-\nu_{n}}{\prod_{j=1}^{n-1} w_{j}}\right) .
$$

Use (3.9) to get

$$
\sqrt{n}\left(\frac{N_{n}(1)}{n}-\frac{\nu_{n}}{n}\right)=\frac{N_{n}-\nu_{n}}{\sqrt{n}} \Rightarrow N\left(0, \sigma_{1}^{2}(\delta)\right),
$$

where $\sigma_{1}(\delta)=\Gamma(1+\gamma) \sigma(\delta)$. Since there exists $K$ such that

$$
\bigvee_{n=1}^{\infty}\left|\nu_{n}-n p_{1}\right| \leqslant K
$$

(eg, 11, Section 8.5]), we may conclude the following.

Proposition 3.1. The number of nodes at stage $n$ with degree 1 is asymptotically normal,

$$
\sqrt{n}\left(\frac{N_{n}(1)}{n}-p_{1}\right) \Rightarrow N\left(0, \sigma_{1}^{2}(\delta)\right)
$$

where

$$
\sigma_{1}^{2}(\delta)=\frac{(1+\delta)(2+\delta)^{2}}{(3+2 \delta)^{2}(4+3 \delta)}
$$

\section{Normality for the Number of nOdes of Degree $k, k>1$.}

The results of Section 3 show how the martingale central limit theorem can be used to prove that the deviation of the fraction of the nodes in the $n$th graph, that have degree 1 , from their limiting fraction, is of the order $n^{-1 / 2}$ and, when normalized by that quantity, has a limiting centered normal distribution; this is the content of Proposition 3.1. It turns out that this distributional result is valid for all node degrees simultaneously. More specifically, a central limit theorem in $\mathbb{R}^{\mathbb{N}}$ holds, and the limit is a centered Gaussian process.

Theorem 4.1. The proportion of nodes with given degrees satisfy the limiting distributional relation

$$
\left(\sqrt{n}\left(\frac{N_{n}(k)}{n}-p_{k}\right), k=1,2, \ldots\right) \Rightarrow\left(Z_{k}, k=1,2 \ldots\right)
$$

in $\mathbb{R}^{\mathbb{N}}$, where $\left(Z_{k}, k=1,2 \ldots\right)$ is a centered Gaussian process with covariance function $R_{Z}$ given by (4.28). 
Remark 4.1. We precede the proof of the theorem with a number of comments.

First of all, weak convergence in $\mathbb{R}^{\mathbb{N}}$ is equivalent to convergence of the finitedimensional distributions; see [1].

It is clear also that, for every fixed $n$, the stochastic process in the left hand side of (4.1) will have at most $n$ random elements; however, all elements in the limiting process are random.

Finally, the variance of $Z_{1}$ in the right hand side of (4.1) is given in Proposition 3.1 .

Proof of Theorem 4.1. As in the one-dimensional case of Section 3 , we will use a martingale central limit theorem, so we start with constructing a suitable martingale for each fixed node degree. For $k=1,2 \ldots$ we denote $\nu_{n}(k)=E\left(N_{n}(k)\right)$, $n \geqslant k$ (so that $\nu_{n}=\nu_{n}(1)$ ). It follows from (3.1) and bounded convergence,

$$
\frac{\nu_{n}(k)}{n} \rightarrow p_{k}, \quad k \geqslant 1 \text {. }
$$

Let

$$
a_{n}^{(k)}=\left[\prod_{i=k}^{n-1}\left(1-\frac{k+\delta}{i(2+\delta)+1+\delta}\right)\right]^{-1}, n \geqslant k,
$$

so that

$$
a_{n+1}^{(k)}=a_{n}^{(k)}\left(1-\frac{k+\delta}{n(2+\delta)+1+\delta}\right)^{-1},
$$

and for future use, note that by the Stirling formula,

$$
\begin{aligned}
a_{n}^{(k)} & =\frac{\Gamma(n+(1+\delta) /(2+\delta)) \Gamma(k+(1-k) /(2+\delta))}{\Gamma(k+(1+\delta) /(2+\delta)) \Gamma(n+(1-k) /(2+\delta))} \\
& \sim \frac{\Gamma(k+(1-k) /(2+\delta))}{\Gamma(k+(1+\delta) /(2+\delta))} n^{(k+\delta) /(2+\delta)} \quad(n \rightarrow \infty)
\end{aligned}
$$

so that as a function of $n, a_{n}^{(k)}$ is regularly varying with index $(k+\delta) /(2+\delta)$. Also define,

$$
b_{j}^{(k)}=\prod_{i=j}^{k-1} \frac{i+\delta}{i-k}=(-1)^{k-j} \frac{\Gamma(k+\delta)}{(k-j) ! \Gamma(j+\delta)}, 1 \leqslant j \leqslant k .
$$

We use the usual conventions to set $a_{k}^{(k)}=b_{k}^{(k)}=1$, and set

$$
M_{n}^{(k)}=a_{n}^{(k)} \sum_{j=1}^{k} b_{j}^{(k)}\left(N_{n}(j)-\nu_{n}(j)\right), n \geqslant k .
$$

The process $\left(M_{n}^{(1)}\right)$ coincides with the process $\left(M_{n}\right)$ defined in (3.6) and we already proved the latter process is a martingale with respect to the filtration $\left(\mathcal{F}_{n}\right)$. Now we check that for each $k \geqslant 1$ the process $\left(M_{n}^{(k)}, n \geqslant k\right)$ is a martingale with respect to the same filtration. 
Recall the dynamics of the of the counting processes $\left(N_{n}(k)\right)$ : for each fixed $n$, there is a partition of the sample space into disjoint events $A_{n}(k), k=0,1, \ldots$, $n \geqslant 1, n \geqslant k$, with

$$
P\left(A_{n}(k) \mid \mathcal{F}_{n}\right)= \begin{cases}\frac{k+\delta}{n(2+\delta)+(1+\delta)} N_{n}(k), & k=1, \ldots, n \\ \frac{1+\delta}{n(2+\delta)+(1+\delta)}, & k=0 .\end{cases}
$$

The event $A_{n}(k)$ is the event that a new node appears at stage $n+1$ and attaches to $v \in V_{n}$ with $D_{n}(v)=k$ while $A_{n}(0)$ is the event that a new node attaches to itself. In terms of these events, for each $k \geqslant 3$ and $n \geqslant k$,

$$
N_{n+1}(k)= \begin{cases}N_{n}(k)+1 & \text { on } A_{n}(k-1), \\ N_{n}(k)-1 & \text { on } A_{n}(k), \\ N_{n}(k) & \text { on } \cup_{j \notin\{k-1, k\}} A_{n}(j),\end{cases}
$$

for $k=2$ and $n \geqslant 2$,

$$
N_{n+1}(2)= \begin{cases}N_{n}(2)+1 & \text { on } A_{n}(0) \cup A_{n}(1), \\ N_{n}(2)-1 & \text { on } A_{n}(2), \\ N_{n}(2) & \text { on } \cup_{j \geqslant 3} A_{n}(j),\end{cases}
$$

while for $k=1$ and $n \geqslant 1$,

$$
N_{n+1}(1)= \begin{cases}N_{n}(1)+1 & \text { on } \cup_{j \geqslant 2} A_{n}(j), \\ N_{n}(1) & \text { on } A_{n}(0) \cup A_{n}(1) .\end{cases}
$$

In particular, for $n \geqslant k$,

$$
\begin{aligned}
E\left(N_{n+1}(k) \mid \mathcal{F}_{n}\right)= & \left(1-\frac{k+\delta}{n(2+\delta)+(1+\delta)}\right) N_{n}(k) \\
& +\frac{k-1+\delta}{n(2+\delta)+(1+\delta)} N_{n}(k-1), k \geqslant 3, \\
E\left(N_{n+1}(2) \mid \mathcal{F}_{n}\right)= & \left(1-\frac{2+\delta}{n(2+\delta)+(1+\delta)}\right) N_{n}(2) \\
& +\frac{1+\delta}{n(2+\delta)+(1+\delta)}\left(1+N_{n}(1)\right) .
\end{aligned}
$$

Taking the expectation, we see that

$$
\begin{aligned}
\nu_{n+1}(k)= & \left(1-\frac{k+\delta}{n(2+\delta)+(1+\delta)}\right) \nu_{n}(k) \\
& +\frac{k-1+\delta}{n(2+\delta)+(1+\delta)} \nu_{n}(k-1), k \geqslant 3, \\
\nu_{n+1}(2)= & \left(1-\frac{2+\delta}{n(2+\delta)+(1+\delta)}\right) \nu_{n}(2) \\
& +\frac{1+\delta}{n(2+\delta)+(1+\delta)}\left(1+\nu_{n}(1)\right) .
\end{aligned}
$$


The corresponding dynamics for $k=1$ is given in (3.3). Therefore, for $n \geqslant k$,

$$
\begin{aligned}
E\left(M_{n+1}^{(k)} \mid \mathcal{F}_{n}\right)= & a_{n+1}^{(k)} \sum_{j=1}^{k} b_{j}^{(k)} E\left[\left(N_{n+1}(j)-\nu_{n+1}(j)\right) \mid \mathcal{F}_{n}\right] \\
= & a_{n+1}^{(k)} b_{1}^{(k)} \frac{n}{n+\gamma}\left(N_{n}(1)-\nu_{n}(1)\right) \\
& +a_{n+1}^{(k)} \sum_{j=2}^{k} b_{j}^{(k)}\left[\left(1-\frac{j+\delta}{n(2+\delta)+(1+\delta)}\right)\left(N_{n}(j)-\nu_{n}(j)\right)\right. \\
& \left.\quad+\frac{j-1+\delta}{n(2+\delta)+(1+\delta)}\left(N_{n}(j-1)-\nu_{n}(j-1)\right)\right] \\
= & a_{n+1}^{(k)}\left\{\sum _ { j = 1 } ^ { k - 1 } \left[b_{j}^{(k)}\left(1-\frac{j+\delta}{n(2+\delta)+(1+\delta)}\right)\right.\right. \\
& \left.+b_{j+1}^{(k)} \frac{j+\delta}{n(2+\delta)+(1+\delta)}\right]\left(N_{n}(j)-\nu_{n}(j)\right) \\
& \left.+b_{k}^{(k)}\left(1-\frac{k+\delta}{n(2+\delta)+(1+\delta)}\right)\left(N_{n}(k)-\nu_{n}(k)\right)\right\}
\end{aligned}
$$

and elementary calculations show that this is the same as the right hand side of (4.7). Therefore for each $k$, the process $\left(M_{n}^{(k)}, n \geqslant k\right)$ is indeed a martingale with respect to the filtration $\left(\mathcal{F}_{n}\right)$.

For $k=1,2, \ldots$ define a $k$-variate triangular array of martingale differences by

$$
X_{n, m, j}=\frac{M_{m}^{(j)}-M_{m-1}^{(j)}}{a_{n}^{(j)} n^{1 / 2}}, m=k+1, \ldots, n, j=1, \ldots, k,
$$

for $n=k, k+1, \ldots$ In order to use the multivariate martingale central limit theorem in Proposition 2.2 we compute the asymptotic form of the quantities

$$
\begin{aligned}
G_{n, m}(i, j): & =E\left(X_{n, m, i} X_{n, m, j} \mid \mathcal{F}_{m-1}\right) \\
& =\left(a_{n}^{(i)} a_{n}^{(j)} n\right)^{-1} E\left(\left(M_{m}^{(i)}-M_{m-1}^{(i)}\right)\left(M_{m}^{(j)}-M_{m-1}^{(j)}\right) \mid \mathcal{F}_{m-1}\right),
\end{aligned}
$$

$m=k+1, \ldots, n, i, j=1, \ldots, k$. By the martingale property,

$$
\begin{aligned}
E( & \left.\left(M_{m+1}^{(i)}-M_{m}^{(i)}\right)\left(M_{m+1}^{(j)}-M_{m}^{(j)}\right) \mid \mathcal{F}_{m}\right) \\
=E & {\left[\sum_{d=1}^{i} b_{d}^{(i)}\left(a_{m+1}^{(i)} N_{m+1}^{(d)}-a_{m}^{(i)} N_{m}^{(d)}\right) \sum_{l=1}^{j} b_{l}^{(j)}\left(a_{m+1}^{(j)} N_{m+1}^{(l)}-a_{m}^{(j)} N_{m}^{(l)}\right) \mid \mathcal{F}_{m}\right] } \\
& \quad-\sum_{d=1}^{i} b_{d}^{(i)}\left(a_{m+1}^{(i)} \nu_{m+1}^{(d)}-a_{m}^{(i)} \nu_{m}^{(d)}\right) \sum_{l=1}^{j} b_{l}^{(j)}\left(a_{m+1}^{(j)} \nu_{m+1}^{(l)}-a_{m}^{(j)} \nu_{m}^{(l)}\right) .
\end{aligned}
$$


We begin by analyzing the behaviour of the deterministic term in the right hand side of (4.18). We claim that for every $i \geqslant 1$,

$$
\lim _{n \rightarrow \infty} \frac{1}{a_{n}^{(i)}} \sum_{d=1}^{i} b_{d}^{(i)}\left(a_{n+1}^{(i)} \nu_{n+1}^{(d)}-a_{n}^{(i)} \nu_{n}^{(d)}\right)=b_{1}^{(i)},
$$

with $b_{1}^{(i)}$ given by (4.6). Indeed, for $d \geqslant 3$, by (4.3) and (4.14) we have

$$
\begin{aligned}
a_{n+1}^{(i)} \nu_{n+1}^{(d)}-a_{n}^{(i)} \nu_{n}^{(d)} & =a_{n+1}^{(i)}\left(\nu_{n}^{(d)} \frac{i-d}{n(2+\delta)+1+\delta}+\nu_{n}^{(d-1)} \frac{d-1+\delta}{n(2+\delta)+1+\delta}\right) \\
& \sim \frac{(i-d) p_{d}+(d-1+\delta) p_{d-1}}{2+\delta} a_{n}^{(i)}
\end{aligned}
$$

as $n \rightarrow \infty$ by (4.2), the fact that $a_{n+1}^{(i)} \sim a_{n}^{(i)}$ and the same is true for $d=2$ by (4.15). Similarly, using (3.3), we obtain for $d=1$ that

$$
a_{n+1}^{(i)} \nu_{n+1}^{(1)}-a_{n}^{(i)} \nu_{n}^{(1)} \sim\left(\frac{i-1}{2+\delta} p_{1}+1\right) a_{n}^{(i)}=a_{n}^{(i)}+a_{n}^{(i)} \frac{i-1}{2+\delta} p_{1},
$$

as $n \rightarrow \infty$. Therefore (with $p_{0}=0$ ),

$$
\begin{aligned}
& \lim _{n \rightarrow \infty} \frac{1}{a_{n}^{(i)}} \sum_{d=1}^{i} b_{d}^{(i)}\left(a_{n+1}^{(i)} \nu_{n+1}^{(d)}-a_{n}^{(i)} \nu_{n}^{(d)}\right) \\
& =b_{1}^{(i)}+\frac{1}{2+\delta} \sum_{d=1}^{i} b_{d}^{(i)}\left[(i-d) p_{d}+(d-1+\delta) p_{d-1}\right]=b_{1}^{(i)},
\end{aligned}
$$

since

$$
\sum_{d=1}^{i} b_{d}^{(i)}\left[(i-d) p_{d}+(d-1+\delta) p_{d-1}\right]=\sum_{d=1}^{i-1} p_{d}\left[(i-d) b_{d}^{(i)}+(d+\delta) b_{d+1}^{(i)}\right]=0 .
$$

Next, by (4.9), (4.10) and (4.11),

$$
a_{n+1}^{(i)} N_{n+1}(d)-a_{n}^{(i)} N_{n}(d)=a_{n+1}^{(i)}\left(N_{n}(d) \frac{i+\delta}{n(2+\delta)+1+\delta}+B_{n}(d)\right),
$$

with

$$
\begin{aligned}
& B_{n}(d)=\left\{\begin{array}{ll}
1 & \text { on } A_{n}(d-1), \\
-1 & \text { on } A_{n}(d), \\
0 & \text { on } \cup_{j \notin\{d-1, d\}} A_{n}(j),
\end{array} \text { for } d \geqslant 3,\right. \\
& B_{n}(2)= \begin{cases}1 & \text { on } A_{n}(0) \cup A_{n}(1), \\
-1 & \text { on } A_{n}(2), \\
0 & \text { on } \cup_{j \geqslant 3} A_{n}(j),\end{cases} \\
& B_{n}(1)= \begin{cases}1 & \text { on } \cup_{j \geqslant 2} A_{n}(j), \\
0 & \text { on } A_{n}(0) \cup A_{n}(1) .\end{cases}
\end{aligned}
$$


RESNICK AND SAMORODNITSKY

Therefore, as $n \rightarrow \infty$,

$$
\begin{aligned}
& E\left[\frac{1}{a_{n}^{(i)} a_{n}^{(j)}} \sum_{d=1}^{i} b_{d}^{(i)}\left(a_{n+1}^{(i)} N_{n+1}(d)-a_{n}^{(i)} N_{n}(d)\right) \sum_{l=1}^{j} b_{l}^{(j)}\left(a_{n+1}^{(j)} N_{n+1}(l)-a_{n}^{(j)} N_{n}(l)\right) \mid \mathcal{F}_{n}\right] \\
& \sim E\left[\sum_{d=1}^{i} b_{d}^{(i)}\left(N_{n}(d) \frac{i+\delta}{n(2+\delta)+1+\delta}+B_{n}(d)\right) \times\right. \\
& \left.\quad \sum_{l=1}^{j} b_{l}^{(j)}\left(N_{n}(l) \frac{j+\delta}{n(2+\delta)+1+\delta}+B_{n}(l)\right) \mid \mathcal{F}_{n}\right] \\
& =\sum_{d=1}^{i} b_{d}^{(i)}(i+\delta) \frac{N_{n}(d)}{n(2+\delta)+1+\delta} \sum_{l=1}^{j} b_{l}^{(j)}(j+\delta) \frac{N_{n}(l)}{n(2+\delta)+1+\delta} \\
& \quad+\sum_{d=1}^{i} b_{d}^{(i)}(i+\delta) \frac{N_{n}(d)}{n(2+\delta)+1+\delta} \sum_{l=1}^{j} b_{l}^{(j)} E\left(B_{n}(l) \mid \mathcal{F}_{n}\right) \\
& \quad+\sum_{l=1}^{j} b_{l}^{(j)}(j+\delta) \frac{N_{n}(l)}{n(2+\delta)+1+\delta} \sum_{d=1}^{i} b_{d}^{(i)} E\left(B_{n}(d) \mid \mathcal{F}_{n}\right) \\
& \quad+\sum_{d=1}^{i} \sum_{l=1}^{j} b_{d}^{(i)} b_{l}^{(j)} E\left(B_{n}(d) B_{n}(l) \mid \mathcal{F}_{n}\right) \\
& :=S_{1, n}(i, j)+S_{2, n}(i, j)+S_{3, n}(i, j)+S_{4, n}(i, j) .
\end{aligned}
$$

It follows by (3.1) that

$$
\sum_{d=1}^{i} b_{d}^{(i)}(i+\delta) \frac{N_{n}(d)}{n(2+\delta)+1+\delta} \rightarrow \frac{i+\delta}{2+\delta} \sum_{d=1}^{i} b_{d}^{(i)} p_{d} \text { a.s. as } n \rightarrow \infty .
$$

Next, by (4.21),

$$
\begin{aligned}
& \sum_{d=1}^{i} b_{d}^{(i)} E\left(B_{n}(d) \mid \mathcal{F}_{n}\right)=b_{1}^{(i)}\left[1-\frac{1+\delta}{n(2+\delta)+1+\delta}\left(1+N_{n}(1)\right)\right] \\
& \quad+\mathbf{1}_{i \geqslant 2} b_{2}^{(i)}\left[\frac{1+\delta}{n(2+\delta)+1+\delta}\left(1+N_{n}(1)\right)-\frac{2+\delta}{n(2+\delta)+1+\delta} N_{n}(2)\right] \\
& \quad+\mathbf{1}_{i} \geqslant 3 \sum_{d=3}^{i} b_{d}^{(i)}\left[\frac{d-1+\delta}{n(2+\delta)+1+\delta} N_{n}(d-1)-\frac{d+\delta}{n(2+\delta)+1+\delta} N_{n}(d)\right] \\
& \rightarrow b_{1}^{(i)}\left(1-\frac{1+\delta}{2+\delta} p_{1}\right)+\mathbf{1}_{i \geqslant 2} \frac{1}{2+\delta} \sum_{d=2}^{i} b_{d}^{(i)}\left((d-1+\delta) p_{d-1}-(d+\delta) p_{d}\right) \\
& =b_{1}^{(i)}+\frac{1}{2+\delta} \sum_{d=1}^{i} b_{d}^{(i)}\left((d-1+\delta) p_{d-1}-(d+\delta) p_{d}\right)
\end{aligned}
$$




$$
=b_{1}^{(i)}-\frac{i+\delta}{2+\delta} \sum_{d=1}^{i} b_{d}^{(i)} p_{d}
$$

a.s., where at the last step we used (4.20). We conclude that, with probability 1 ,

$$
\begin{aligned}
& S_{1, n}(i, j) \rightarrow \frac{(i+\delta)(j+\delta)}{(2+\delta)^{2}} \sum_{d=1}^{i} b_{d}^{(i)} p_{d} \sum_{l=1}^{j} b_{l}^{(j)} p_{l}, \\
& S_{2, n}(i, j) \rightarrow \frac{i+\delta}{2+\delta} \sum_{d=1}^{i} b_{d}^{(i)} p_{d}\left(b_{1}^{(j)}-\frac{j+\delta}{2+\delta} \sum_{l=1}^{j} b_{l}^{(j)} p_{l}\right), \\
& S_{3, n}(i, j) \rightarrow \frac{j+\delta}{2+\delta} \sum_{l=1}^{i} j b_{l}^{(j)} p_{l}\left(b_{1}^{(i)}-\frac{i+\delta}{2+\delta} \sum_{d=1}^{i} b_{d}^{(i)} p_{d}\right) .
\end{aligned}
$$

Finally, we consider the term $S_{4, n}(i, j)$. Note that, by (4.21), we have the following cases.

(1) On $A_{n}(0) \cup A_{n}(1)$ :

$$
E\left(B_{n}(d) B_{n}(l) \mid \mathcal{F}_{n}\right)=\mathbf{1}_{d=l=2} .
$$

(2) On $A_{n}(m), m \geqslant 2$,

$$
E\left(B_{n}(d) B_{n}(l) \mid \mathcal{F}_{n}\right)= \begin{cases}1 & \text { if } d, l \in\{1, m+1\} \text { or } d=l=m \\ -1 & \text { if } d=m, l \in\{1, m+1\} \text { or } l=m, d \in\{1, m+1\}\end{cases}
$$

Therefore, using the convention $b_{d}^{(i)}=0$ if $d>i$, we can write

$$
\begin{aligned}
& S_{4, n}(i, j)=b_{2}^{(i)} b_{2}^{(j)} \frac{1+\delta}{n(2+\delta)+1+\delta}\left(1+N_{n}^{(1)}\right) \\
& +\sum_{m=2}^{n}\left(b_{1}^{(i)}-b_{m}^{(i)}+b_{m+1}^{(i)}\right)\left(b_{1}^{(j)}-b_{m}^{(j)}+b_{m+1}^{(j)}\right) \frac{m+\delta}{n(2+\delta)+1+\delta} N_{n}^{(m)} \\
& \rightarrow \sum_{m=1}^{\infty} \frac{m+\delta}{2+\delta}\left(b_{1}^{(i)}-b_{m}^{(i)}+b_{m+1}^{(i)}\right)\left(b_{1}^{(j)}-b_{m}^{(j)}+b_{m+1}^{(j)}\right) p_{m}
\end{aligned}
$$

a.s. as $n \rightarrow \infty$. We conclude that, with probability 1 ,

$$
\begin{gathered}
E\left[\frac{1}{a_{n}^{(i)} a_{n}^{(j)}}\left(M_{n+1}^{(i)}-M_{n}^{(i)}\right)\left(M_{n+1}^{(j)}-M_{n}^{(j)}\right) \mid \mathcal{F}_{n}\right] \rightarrow a(i, j) \\
=: \sum_{m=1}^{\infty} \frac{m+\delta}{2+\delta}\left(b_{1}^{(i)}-b_{m}^{(i)}+b_{m+1}^{(i)}\right)\left(b_{1}^{(j)}-b_{m}^{(j)}+b_{m+1}^{(j)}\right) p_{m} \\
\quad-\left(b_{1}^{(i)}-\frac{i+\delta}{2+\delta} \sum_{d=1}^{i} b_{d}^{(i)} p_{d}\right)\left(b_{1}^{(j)}-\frac{j+\delta}{2+\delta} \sum_{l=1}^{j} b_{l}^{(j)} p_{l}\right) .
\end{gathered}
$$


Next we simplify the expression for $a(i, j)$, to make it clear that $a(i, i)>0$ for all $i \geqslant 1$. Note that, by (3.2) and (4.6), for each $i$,

$$
\begin{aligned}
& \sum_{d=1}^{i-1} b_{d}^{(i)} p_{d}=c(\delta)(-1)^{i} \Gamma(i+\delta) \sum_{d=1}^{i-1}(-1)^{d} \frac{1}{(i-d) ! \Gamma(d+3+2 \delta)} \\
& =c(\delta) \frac{(-1)^{i} \Gamma(i+\delta)}{i+2+2 \delta} \sum_{d=1}^{i-1}(-1)^{d}\left[\frac{1}{(i-d) ! \Gamma(d+2+2 \delta)}+\frac{1}{(i-d-1) ! \Gamma(d+3+2 \delta)}\right] \\
& =c(\delta) \frac{(-1)^{i} \Gamma(i+\delta)}{i+2+2 \delta}\left(-\frac{1}{(i-1) ! \Gamma(3+2 \delta)}+(-1)^{i-1} \frac{1}{\Gamma(i+2+2 \delta)}\right),
\end{aligned}
$$

where at the last step we used the telescoping property of the sum. Elementary algebra now gives us for $i \geqslant 1$,

$$
b_{1}^{(i)}-\frac{i+\delta}{2+\delta} \sum_{d=1}^{i} b_{d}^{(i)} p_{d}=\frac{2+\delta}{\Gamma(1+\delta)} \frac{(-1)^{i-1} \Gamma(i+\delta)}{(i-1) ! \Gamma(i+2+2 \delta)} .
$$

Similarly, for $i \geqslant 1$, using a telescopic property,

$$
\begin{aligned}
& \sum_{m=i+1}^{\infty} \frac{m+\delta}{2+\delta}\left(b_{1}^{(i)}-b_{m}^{(i)}+b_{m+1}^{(i)}\right)^{2} p_{m}=\left(b_{1}^{(i)}\right)^{2} \frac{c(\delta)}{2+\delta} \sum_{m=i+1}^{\infty} \frac{\Gamma(m+1+\delta)}{\Gamma(m+3+2 \delta)} \\
= & \left(b_{1}^{(i)}\right)^{2} \frac{c(\delta)}{2+\delta} \sum_{m=i+1}^{\infty} \frac{1}{1+\delta}\left[\frac{\Gamma(m+1+\delta)}{\Gamma(m+2+2 \delta)}-\frac{\Gamma(m+2+\delta)}{\Gamma(m+3+2 \delta)}\right] \\
= & \left(b_{1}^{(i)}\right)^{2} \frac{c(\delta)}{(1+\delta)(2+\delta)} \frac{\Gamma(i+2+\delta)}{\Gamma(i+3+2 \delta)} \\
= & \frac{\Gamma(3+2 \delta)}{\Gamma(2+\delta)(\Gamma(1+\delta))^{2}} \frac{\Gamma(i+2+\delta)(\Gamma(i+\delta))^{2}}{\Gamma(i+3+2 \delta)((i-1) !)^{2}}
\end{aligned}
$$

Therefore, by (4.23),

$$
\begin{aligned}
& a(i, i)>\sum_{m=i+1}^{\infty} \frac{m+\delta}{2+\delta}\left(b_{1}^{(i)}-b_{m}^{(i)}+b_{m+1}^{(i)}\right)^{2} p_{m}-\left(b_{1}^{(i)}-\frac{i+\delta}{2+\delta} \sum_{d=1}^{i} b_{d}^{(i)} p_{d}\right)^{2} \\
& =\left(\frac{2+\delta}{\Gamma(1+\delta)} \frac{\Gamma(i+\delta)}{(i-1) !(i+2+2 \delta)}\right)^{2}\left[\frac{\Gamma(3+2 \delta)}{(2+\delta) \Gamma(3+\delta)} \frac{(i+2+2 \delta) \Gamma(i+2+\delta)}{\Gamma(i+2+2 \delta)}-1\right] .
\end{aligned}
$$

Note that the expression inside the bracket is greater than

$$
\frac{\Gamma(3+2 \delta)}{(2+\delta) \Gamma(3+\delta)} \frac{\Gamma(i+2+\delta)}{\Gamma(i+1+2 \delta)}-1,
$$

and, since for $0<b<a$, the ratio $\Gamma(x+a) / \Gamma(x+b)$ is increasing in $x \geqslant 0$, the above difference is, for $i \geqslant 2$, at least

$$
\frac{\Gamma(3+2 \delta)}{(2+\delta) \Gamma(3+\delta)} \frac{\Gamma(2+2+\delta)}{\Gamma(2+1+2 \delta)}-1=\frac{3+\delta}{2+\delta}-1>0 .
$$


This shows that $a(i, i)>0$ for all $i \geqslant 2$. The fact that the same is true for $i=1$ can be seen directly from (4.22) (and was also shown in Section 3).

We know from (4.22) that

$$
n G_{n, n}(i, j) \rightarrow a(i, j), \quad(n \rightarrow \infty),
$$

and from the definition (4.17) we have

$$
G_{n, m}=\frac{a_{m}^{(i)} a_{m}^{(j)}}{n a_{n}^{(i)} a_{n}^{(i)}} m G_{m, m}(i, j)
$$

and from the regular variation property after (4.5), the function

$$
u(m):=a_{m}^{(i)} a_{m}^{(i)} m G_{m, m}(i, j)
$$

is regularly varying with index $(i+j+2 \delta) /(2+\delta)$. Therefore, from Karamata's theorem on integration of regularly varying functions

$$
\begin{aligned}
V_{n}(i, j) & =\sum_{m=k+1}^{n} G_{n, m}(i, j)=\frac{\sum_{m=k}^{n} u(m)}{n a_{n}^{(i)} a_{n}^{(j)}} \\
& \sim \frac{n u(n)}{\left(\frac{i+j+2 \delta}{2+\delta}+1\right) n a_{n}^{(i)} a_{n}^{(i)}} \sim a(i, j) \frac{2+\delta}{i+j+3 \delta+2},
\end{aligned}
$$

for $i, j=1, \ldots, k$, where $a(i, j)$ is defined in (4.22). This verifies the first condition the martingale central limit theorem of Proposition 2.2 (with each $A_{n}$ being a $k \times k$ identity matrix.) The second condition of the theorem holds as well, since by (4.9), the differences are bounded,

$$
\left|\left(N_{n}(j)-\nu_{n}(j)\right)-\left(N_{n-1}(j)-\nu_{n-1}(j)\right)\right| \leqslant 2 \text { for all } j,
$$

hence, as in the one-dimensional case of Section 3, for all $n$ large enough, the events $\left\{X_{n, m, j}>\epsilon\right\}$ are empty for all $m \leqslant n$ and all $j$. We conclude that

$$
\left(\frac{1}{n^{1 / 2}} \sum_{j=1}^{k} b_{j}^{(k)}\left(N_{n}(j)-\nu_{n}(j)\right), k \geqslant 1\right) \Rightarrow\left(Y_{k}, k=1,2 \ldots\right)
$$

in $\mathbb{R}^{\mathbb{N}}$, where $\left(Y_{k}, k=1,2 \ldots\right)$ is a centered Gaussian process with covariance function $R_{Y}$ given by

$$
R_{Y}(i, j)=\frac{2+\delta}{i+j+2+3 \delta} a(i, j), i, j \geqslant 1 .
$$

We use this covariance function to define the $k \times k$ matrix

$$
R_{Y, k}=\left(R_{Y}(i, j), 1 \leqslant i, j \leqslant k\right) .
$$

For a fixed $k=1,2 \ldots$ the convergence in (4.24) means that

$$
C_{k}\left(\frac{N_{n}(j)-\nu_{n}(j)}{n^{1 / 2}}, j=1, \ldots, k\right)^{T} \Rightarrow\left(Y_{j}, j=1, \ldots, k\right),
$$


where $C_{k}$ is a $k \times k$ matrix with the entries

$$
c_{i, j}= \begin{cases}b_{j}^{(i)} & j \leqslant i \\ 0 & j>i\end{cases}
$$

Using the easily checkable identity, valid for any real $r$,

$$
\sum_{m=j}^{i} r^{m-j} b_{m}^{(i)} b_{j}^{(m)}=b_{j}^{(i)}(1+r)^{i-j}, 1 \leqslant j \leqslant i,
$$

we can check that the inverse of $C_{k}, D_{k}=C_{k}^{-1}$, has the entries

$$
d_{i, j}= \begin{cases}(-1)^{i-j} b_{j}^{(i)} & j \leqslant i \\ 0 & j>i\end{cases}
$$

We conclude that

$$
\left(\frac{N_{n}(j)-\nu_{n}(j)}{n^{1 / 2}}, j=1, \ldots, k\right) \Rightarrow D_{k}\left(Y_{j}, j=1, \ldots, k\right)^{T},
$$

and the covariance matrix of the limiting Gaussian vector is given by

$$
\Sigma_{k}=D_{k} R_{Y, k} D_{k}^{T},
$$

where $R_{Y, k}$ is the $k \times k$ matrix given after (4.25).

In order to facilitate the calculation of the entries of the matrix $\Sigma_{k}$, we write the matrix $R_{Y, k}$ in the form

$$
R_{Y, k}=\sum_{m=0}^{\infty} h_{m} \int_{0}^{\infty}(2+\delta) e^{-(2+3 \delta) x} R_{m, x} d x
$$

where

$$
h_{m}= \begin{cases}-1 & m=0, \\ \frac{m+\delta}{2+\delta} p_{m} & m=1,2, \ldots,\end{cases}
$$

and the matrix $R_{m, x}$ is a $k \times k$ matrix of the form

$$
R_{m, x}=C_{m, x}^{T} \boldsymbol{C}_{m, x} .
$$

Here $\boldsymbol{C}_{m, x}$ is a vector with the entries

$$
C_{m, x}(i)= \begin{cases}\left(b_{1}^{(i)}-\frac{i+\delta}{2+\delta} \sum_{d=1}^{i} b_{d}^{(i)} p_{d}\right) e^{-i x}, i \geqslant 1 & m=0 \\ \left(b_{1}^{(i)}-b_{m}^{(i)}+b_{m+1}^{(i)}\right) e^{-i x}, i \geqslant 1 & m \geqslant 1 .\end{cases}
$$

Therefore,

$$
\Sigma_{k}=\sum_{m=0}^{\infty} h_{m} \int_{0}^{\infty}(2+\delta) e^{-(2+3 \delta) x} D_{k} \boldsymbol{C}_{m, x}^{T} \boldsymbol{C}_{m, x} D_{k}^{T} d x .
$$

Note that by (4.27) and (4.26), for any $m \geqslant 1$ and $i=1,2, \ldots$,

$$
\begin{gathered}
\left(D_{k} \boldsymbol{C}_{m, x}^{T}\right)(i)=\sum_{j=1}^{i}(-1)^{i-j} b_{j}^{(i)}\left(b_{1}^{(j)}-b_{m}^{(j)}+b_{m+1}^{(j)}\right) e^{-j x} \\
=e^{-x} b_{1}^{(i)}\left(-1+e^{-x}\right)^{i-1}-e^{-m x} b_{m}^{(i)}\left(-1+e^{-x}\right)^{i-m}+e^{-(m+1) x} b_{m+1}^{(i)}\left(-1+e^{-x}\right)^{i-m-1} .
\end{gathered}
$$


Therefore, for $m \geqslant 1$ and $i, j=1,2, \ldots$,

$$
\begin{aligned}
\left(D_{k} \boldsymbol{C}_{m, x}^{T}\right)(i)\left(D_{k} \boldsymbol{C}_{m, x}^{T}\right)(j) & =b_{1}^{(i)} b_{1}^{(j)} e^{-2 x}\left(-1+e^{-x}\right)^{i+j-2} \\
& -\left(b_{1}^{(i)} b_{m}^{(j)}+b_{m}^{(i)} b_{1}^{(j)}\right) e^{-(m+1) x}\left(-1+e^{-x}\right)^{i+j-m-1} \\
& +\left(b_{1}^{(i)} b_{m+1}^{(j)}+b_{m+1}^{(i)} b_{1}^{(j)}\right) e^{-(m+2) x}\left(-1+e^{-x}\right)^{i+j-m-2} \\
& +b_{m}^{(i)} b_{m}^{(j)} e^{-2 m x}\left(-1+e^{-x}\right)^{i+j-2 m} \\
& -\left(b_{m}^{(i)} b_{m+1}^{(j)}+b_{m+1}^{(i)} b_{m}^{(j)}\right) e^{-(2 m+1) x}\left(-1+e^{-x}\right)^{i+j-2 m-1} \\
& +b_{m+1}^{(i)} b_{m+1}^{(j)} e^{-(2 m+2) x}\left(-1+e^{-x}\right)^{i+j-2 m-2} \\
& :=\sum_{l=1}^{6} \theta_{m, x}^{(l)}(i, j) .
\end{aligned}
$$

We have:

$$
\begin{aligned}
& \int_{0}^{\infty}(2+\delta) e^{-(2+3 \delta) x} \theta_{m, x}^{(1)}(i, j) d x \\
& =(-1)^{i+j}(2+\delta) b_{1}^{(i)} b_{1}^{(j)} \int_{0}^{\infty} e^{-(4+3 \delta) x}\left(1-e^{-x}\right)^{i+j-2} d x \\
& =(-1)^{i+j}(2+\delta) b_{1}^{(i)} b_{1}^{(j)} B(4+3 \delta, i+j-1) \\
& =(-1)^{i+j}(2+\delta) b_{1}^{(i)} b_{1}^{(j)} \frac{\Gamma(4+3 \delta)(i+j-2) !}{\Gamma(i+j+3+3 \delta)}
\end{aligned}
$$

Similarly,

$$
\begin{aligned}
& \int_{0}^{\infty}(2+\delta) e^{-(2+3 \delta) x} \theta_{m, x}^{(2)}(i, j) d x \\
& =(-1)^{i+j-m}(2+\delta)\left(b_{1}^{(i)} b_{m}^{(j)}+b_{m}^{(i)} b_{1}^{(j)}\right) \frac{\Gamma(m+3+3 \delta)(i+j-m-1) !}{\Gamma(i+j+3+3 \delta)} \\
& \int_{0}^{\infty}(2+\delta) e^{-(2+3 \delta) x} \theta_{m, x}^{(3)}(i, j) d x \\
& =(-1)^{i+j-m}(2+\delta)\left(b_{1}^{(i)} b_{m+1}^{(j)}+b_{m+1}^{(i)} b_{1}^{(j)}\right) \frac{\Gamma(m+4+3 \delta)(i+j-m-2) !}{\Gamma(i+j+3+3 \delta)} \\
& \quad \int_{0}^{\infty}(2+\delta) e^{-(2+3 \delta) x} \theta_{m, x}^{(4)}(i, j) d x \\
& \quad=(-1)^{i+j}(2+\delta) b_{m}^{(i)} b_{m}^{(j)} \frac{\Gamma(2 m+2+3 \delta)(i+j-2 m) !}{\Gamma(i+j+3+3 \delta)} \\
& \int_{0}^{\infty}(2+\delta) e^{-(2+3 \delta) x} \theta_{m, x}^{(5)}(i, j) d x \\
& =(-1)^{i+j}(2+\delta)\left(b_{m}^{(i)} b_{m+1}^{(j)}+b_{m+1}^{(i)} b_{m}^{(j)}\right) \frac{\Gamma(2 m+3+3 \delta)(i+j-2 m-1) !}{\Gamma(i+j+3+3 \delta)}
\end{aligned}
$$




$$
\begin{aligned}
& \int_{0}^{\infty}(2+\delta) e^{-(2+3 \delta) x} \theta_{m, x}^{(6)}(i, j) d x \\
& =(-1)^{i+j}(2+\delta) b_{m+1}^{(i)} b_{m+1}^{(j)} \frac{\Gamma(2 m+4+3 \delta)(i+j-2 m-2) !}{\Gamma(i+j+3+3 \delta)}
\end{aligned}
$$

Similarly, by (4.27) and (4.23), for $i \geqslant 1$,

$$
\left(D_{k} \boldsymbol{C}_{0, x}^{T}\right)(i)=\frac{(2+\delta) \Gamma(i+\delta)}{\Gamma(1+\delta)} \sum_{l=1}^{i}(-1)^{l-1} e^{-l x} \frac{1}{(i-l) !(l-1) ! \Gamma(l+2+2 \delta)} .
$$

Therefore, for $i, j \geqslant 1$,

$$
\begin{gathered}
\int_{0}^{\infty}(2+\delta) e^{-(2+3 \delta) x}\left(D_{k} \boldsymbol{C}_{0, x}^{T}\right)(i)\left(\boldsymbol{C}_{0, x} D_{k}^{T}\right)(j) d x \\
=\frac{(2+\delta)^{2} \Gamma(i+\delta) \Gamma(j+\delta)}{(\Gamma(1+\delta))^{2}} \\
\sum_{l_{1}=1}^{i} \sum_{l_{2}=1}^{j} \frac{(-1)^{l_{1}+l_{2}}\left(l_{1}+l_{2}+2+3 \delta\right)^{-1}}{\left(i-l_{1}\right) !\left(l_{1}-1\right) ! \Gamma\left(l_{1}+2+2 \delta\right)\left(j-l_{2}\right) !\left(l_{2}-1\right) ! \Gamma\left(l_{2}+2+2 \delta\right)} .
\end{gathered}
$$

We conclude that the covariance function of the limiting Gaussian process $\left(Z_{k}, k=\right.$ $1,2 \ldots)$ in (4.1) is given by

$$
\begin{aligned}
R_{Z}(i, j) & =\frac{(-1)^{i+j}}{\Gamma(i+j+3+3 \delta)} \\
4.28) \quad & \sum_{m=1}^{\infty}(m+\delta) p_{m}\left[b_{1}^{(i)} b_{1}^{(j)} \Gamma(4+3 \delta)(i+j-2) !\right. \\
& +(-1)^{m}\left(b_{1}^{(i)} b_{m}^{(j)}+b_{m}^{(i)} b_{1}^{(j)}\right) \Gamma(m+3+3 \delta)(i+j-m-1) ! \\
& +(-1)^{m}\left(b_{1}^{(i)} b_{m+1}^{(j)}+b_{m+1}^{(i)} b_{1}^{(j)}\right) \Gamma(m+4+3 \delta)(i+j-m-2) ! \\
& +b_{m}^{(i)} b_{m}^{(j)} \Gamma(2 m+2+3 \delta)(i+j-2 m) ! \\
& +\left(b_{m}^{(i)} b_{m+1}^{(j)}+b_{m+1}^{(i)} b_{m}^{(j)}\right) \Gamma(2 m+3+3 \delta)(i+j-2 m-1) ! \\
& \left.+b_{m+1}^{(i)} b_{m+1}^{(j)} \Gamma(2 m+4+3 \delta)(i+j-2 m-2) !\right] \\
& -\frac{(2+\delta)^{2} \Gamma(i+\delta) \Gamma(j+\delta)}{(\Gamma(1+\delta))^{2}} \\
& \sum_{l_{1}=1}^{i} \sum_{l_{2}=1}^{j} \frac{(-1)^{l_{1}+l_{2}}\left(l_{1}+l_{2}+2+3 \delta\right)^{-1}}{\left(i-l_{1}\right) !\left(l_{1}-1\right) ! \Gamma\left(l_{1}+2+2 \delta\right)\left(j-l_{2}\right) !\left(l_{2}-1\right) ! \Gamma\left(l_{2}+2+2 \delta\right)}
\end{aligned}
$$

Once again, it is possible to show that $R_{Z}(i, i)>0$ for all $i \geqslant 1$. We omit the argument. 


\section{Concluding Remarks}

The model considered here is relatively simple and the calculations are relatively complex and it remains to be seen which more realistic models allow us to successfully conclude asymptotic normality of degree counts. We are particularly anxious to extend our methods to directed graphs where each node is indexed by (at least) two characteristics such as in and out degree. Some investigations are currently underway for the directed preferential attachment model considered in [3, 7, 9, 10]. We also have a program evaluating various inferential methods for estimating model parameters which requires asymptotic normality results such as presented here.

\section{REFERENCES}

[1] P. Billingsley. Convergence of Probability Measures. John Wiley \& Sons Inc., New York, 1968.

[2] N. H. Bingham, C. M. Goldie, and J. L. Teugels. Regular Variation, volume 27 of Encyclopedia of Mathematics and its Applications. Cambridge University Press, Cambridge, 1989. ISBN 0-521-37943-1.

[3] B. Bollobás, C. Borgs, J. Chayes, and O. Riordan. Directed scale-free graphs. In Proceedings of the Fourteenth Annual ACM-SIAM Symposium on Discrete Algorithms (Baltimore, 2003), pages 132-139, New York, 2003. ACM.

[4] R. Durrett. Probability: Theory and Examples. Cambridge Series in Statistical and Probabilistic Mathematics. Cambridge University Press, Cambridge, fourth edition, 2010. ISBN 978-0-521-76539-8. doi: 10.1017/CBO9780511779398. URL http://dx.doi.org/10.1017/CB09780511779398.

[5] R.T. Durrett. Random Graph Dynamics. Cambridge Series in Statistical and Probabilistic Mathematics. Cambridge University Press, Cambridge, 2010. ISBN 978-0-521-15016-3.

[6] P. Hall and C. C. Heyde. Martingale Limit Theory and its Application. Academic Press Inc. [Harcourt Brace Jovanovich Publishers], New York, 1980. ISBN 0-12-319350-8. Probability and Mathematical Statistics.

[7] P.L. Krapivsky and S. Redner. Organization of growing random networks. Physical Review E, 63(6):066123:1-14, 2001.

[8] S.I. Resnick. Heavy Tail Phenomena: Probabilistic and Statistical Modeling. Springer Series in Operations Research and Financial Engineering. SpringerVerlag, New York, 2007. ISBN: 0-387-24272-4.

[9] S.I. Resnick and G. Samorodnitsky. Tauberian Theory for Multivariate Regularly Varying Distributions with Application to Preferential Attachment Networks. Extremes, 2015. doi: 10.1007/s10687-015-0216-2. URL http://adsabs.harvard.edu/abs/2014arXiv1406.6395R.

[10] G. Samorodnitsky, S. Resnick, D. Towsley, R. Davis, A. Willis, and P. Wan. Nonstandard regular variation of in-degree and out-degree in the preferential attachment model. Journal of Applied Probability, 53(1), March 2016. http://arxiv.org/pdf/1405.4882.pdf 
[11] R. van der Hofstad. Random graphs and complex networks, 2014. Available at http://www.win.tue.nl/ rhofstad/; 300 pages.

Sidney I. Resnick, School of ORIE, Cornell University, IthacA, NY 14853

E-mail address: sir1@cornell.edu

Gennady Samorodnitsky, School of ORIE, Cornell University, Ithaca, Ny 14853

E-mail address: gs18@cornell.edu 